\title{
Fast Wavelength Switching 112Gb/s Coherent Burst Mode Transceiver for Dynamic Optical Networks
}

\author{
Robert Maher $^{(1,2)}$, David S. Millar ${ }^{(1)}$, Seb J. Savory ${ }^{(1)}$, Benn C. Thomsen ${ }^{(1)}$
}

(1) Optical Networks Group, Department of Electronic and Electrical Engineering, UCL, Torrington Place, London, UK, r.maher@ee.ucl.ac.uk

(2) The RINCE Institute, School of Electronic Engineering, Dublin City University, Glasnevin, Dublin 9, Ireland

Abstract Using commercially available semiconductor tunable lasers with SOA blanking and preemphasis, we demonstrate fast synchronous switching of a multichannel $112 \mathrm{~Gb} / \mathrm{s}$ DP-QPSK burst mode transceiver in an 8-channel $240 \mathrm{~km}$ DWDM optical link with 200ns reconfiguration time.

\section{Introduction}

As data traffic demands increase there has been renewed interest in fast switching optical networks that provide reconfigurable subwavelength bandwidth allocation for applications such as HPC and data center networks [1]. Previous optical networks employing dynamic circuit or packet switching have focused on either a fast tunable transmitter with fixed receivers [2] or fixed transmitters with a tunable receiver [3]. Here we demonstrate for the first time, to the best of our knowledge, a complete $112 \mathrm{~Gb} / \mathrm{s}$ DP-QPSK coherent burst mode transceiver that provides fast wavelength switching at both the transmitter and receiver using commercially available wavelength tunable digital supermode distributed Bragg reflector (DS-DBR) lasers [4].

The inherent frequency selectivity of digital coherent reception, combined with fast wavelength switching DS-DBR lasers within each network transmit and receive node, provides the channel selectivity required to maximize the efficiency and flexibility of networks that require sub-wavelength dynamic bandwidth allocation at the optical layer.

However, digital coherent reception places strict requirements on the frequency and phase stability of both the transmitter and receiver tunable lasers when under switched operation. For coherent burst mode optical networks the transceiver must be reconfigured within $\sim 200 \mathrm{~ns}$ [5], where the emission frequency of the transmitter and local oscillator (LO) lasers must be within $\pm 1 / 8$ of the signal Baud rate. Therefore energy efficient, integrated widely tunable (Cband) semiconductor lasers that exhibit low phase noise and switching times less than 100ns must be utilized.

We characterize the performance of a $112 \mathrm{~Gb} / \mathrm{s}$ burst mode transceiver in an 8channel, 240km DWDM transmission link that contains both static (continuous) and dynamic wavelength switched (burst) channels with a network reconfiguration time of less than 200ns. Additionally, we show that it is necessary to use pre-emphasis on the rear and phase tuning sections of the transmitter DS-DBR laser, when blanking the integrated SOA during a switching event. This compensates for the wavelength drift that is induced by the large current swing applied to the SOA and ensures that wavelength switching of the transmitter does not interfere with adjacent DWDM channels.

\section{Coherent Burst Mode Transceiver}

The DWDM test-bed, shown in Fig. 1, used eight channels on a $50 \mathrm{GHz}$ grid. Four $100 \mathrm{GHz}$ spaced (odd) continuous channels were provided by an array of DFB lasers and were interleaved with four (even) $100 \mathrm{GHz}$ spaced dynamic channels with a burst length of $5 \mu \mathrm{s}$, which were provided by a single DS-DBR laser. All ten current tuning sections of the DS-DBR laser and the integrated SOA were directly driven from a $250 \mathrm{MS} / \mathrm{s}$ arbitrary waveform generator, which allowed it to be rapidly tuned (within 100ns) over 89 channels within the Cband on a $50 \mathrm{GHz}$ grid.

The odd and even channels were combined together in a $3 \mathrm{~dB}$ coupler and the two outputs

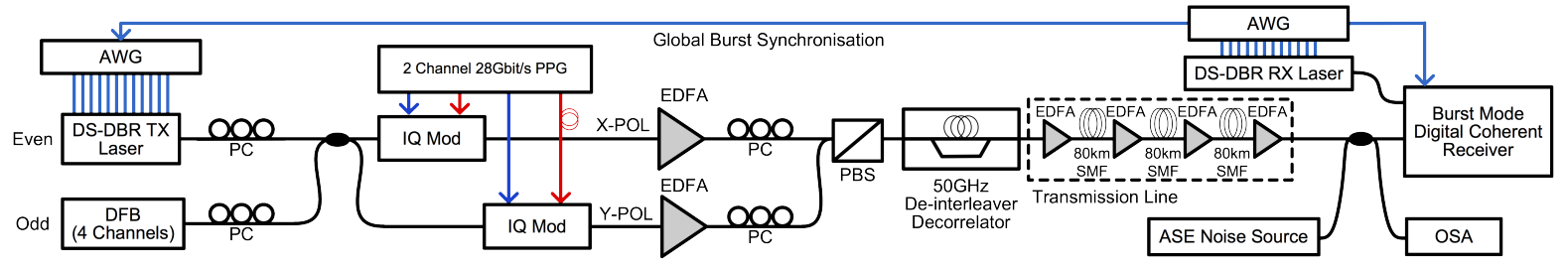

Fig. 1: $112 \mathrm{~Gb} / \mathrm{s}$ DP-QPSK burst mode transceiver and 240km DWDM transmission test bed. 
were sent to two identical IQ modulators, one for each polarization. The I and $\mathrm{Q}$ components were driven with two decorrelated $28 \mathrm{~Gb} / \mathrm{s}$ signals, derived from two independently interleaved $14 \mathrm{~Gb} / \mathrm{s} 2^{15}-1$ PRBS data streams. This generated a $28 \mathrm{Gbaud}$ QPSK signal on the $X$ and $Y$-polarizations, which were subsequently passed through a polarization beam combiner to provide an 8-channel $112 \mathrm{~Gb} / \mathrm{s}$ DP-QPSK signal. The wavelength channels were decorrelated using a $50 \mathrm{GHz}$ interleaver, which had a fiber delay (680 symbols) in one arm. The $240 \mathrm{~km}$ transmission line consisted of three $80 \mathrm{~km}$ spans of SMF with a dispersion of $16.8 \mathrm{ps} / \mathrm{nm} . \mathrm{km}$ at $1554 \mathrm{~nm}$. Each fiber span was followed by an EDFA, which provided a launch power of approximately $0 \mathrm{dBm}$ per channel.

A second DS-DBR laser, driven in an identical fashion to the transmitter, was used as the LO in the coherent burst mode receiver. The LO frequency could be tuned to coincide with either a single channel or synchronously switched to cycle through the incoming burst and continuous channels. The side mode suppression ratio for each channel was greater than $45 \mathrm{~dB}$ and the output power was $12 \mathrm{dBm}$. The transmitted channels were coherently detected and processed offline using Matlab, which incorporated the burst mode parallel DSP receiver implementation (including digital dispersion compensation) described in [6].

\section{Switching Signal Pre-emphasis}

Initially, no modulation was applied to the DSDBR transmitter laser (DFB lasers were unbiased) in order to analyze the switching characteristics and signal pre-emphasis. Fig. 2(a) illustrates the required switching voltages applied to the rear, phase and SOA sections of the DS-DBR laser to sequentially switch between the four $5 \mu$ s burst channels. The laser output must be blanked during tuning to avoid the transmission of spurious modes, thereby mitigating the impact of cross channel interference in a DWDM network. Therefore the SOA was turned off (by applying a small reverse bias) $25 \mathrm{~ns}$ before the switching voltages were applied to the tuning sections of the DS-DBR laser and then turned back on 50ns after the switch, as seen in the inset of Fig. 2(a).

Although adequate spurious mode suppression $(50 \mathrm{~dB})$ is achieved by rapidly turning the SOA on and off, the associated current swing applied to the SOA causes a time dependent $50 \mathrm{GHz}$ mode hop to lower frequencies, as seen in Fig. 2(b). This mode hop will also cause cross channel interference in a DWDM network and thus degrade system performance. To avoid a mode hop, pre- emphasis was applied to the rear and phase tuning sections of the DS-DBR laser.

The signal was pre-emphasized over a short time period, as seen inset of Fig. 2(c), to ensure that the laser frequency stayed within the selected channel. Fig. 2(d) shows that when signal pre-emphasis is employed, the mode hop resulting from SOA blanking has been compensated.
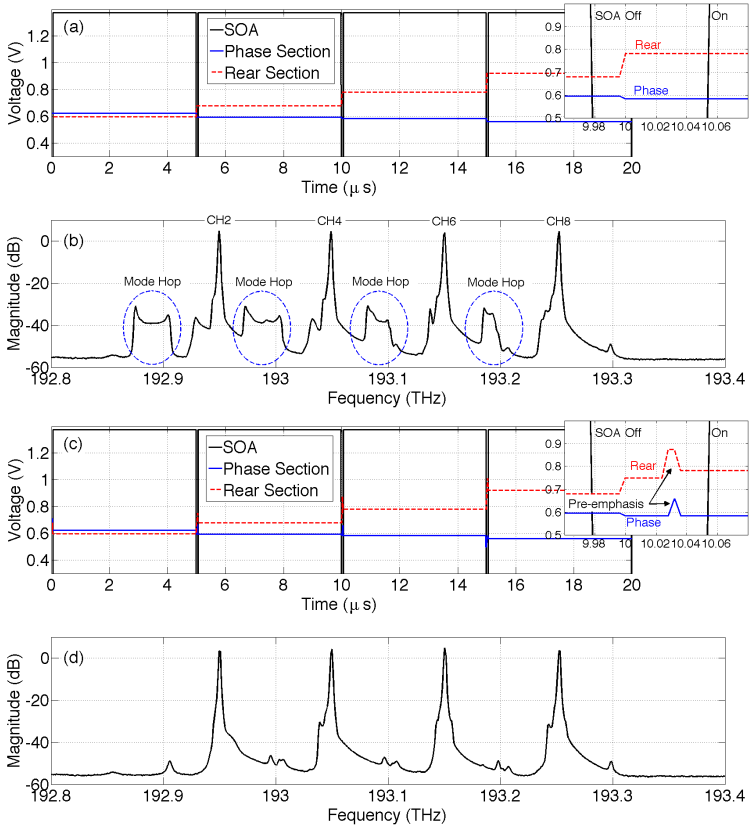

Fig. 2. (a) Switching signals applied to DS-DBR laser, (b) four $5 \mu$ s burst channels with SOA blanking but without preemphasis, (c) switching signals with pre-emphasis (d) four $5 \mu$ s burst channels with SOA blanking and pre-emphasis.

\section{Results and Discussion}

Fig. 3(a) illustrates the switching map of the four odd continuous channels and two cycles of the four even burst channels at the transmitter. The LO could be tuned either statically to permanently reside at a continuous channel or sequentially switch to first cycle through the continuous and then the burst channels as seen in Fig. 3(a). Fig. 3(b) shows the time resolved differentially decoded BER performance for each continuous channel when pre-emphasis was and was not applied to the transmitter DSDBR laser. The BER is estimated over a block length of 8192 bits to show the time dynamics and provide an accurate measure of the BER at a FEC limit of $3.8 \times 10^{-3}$. It is necessary to use differential decoding in the receiver to minimize the impact of cycle slips that arise from the combined linewidths of the transmitter and LO DS-DBR lasers under dynamic operation (DSDBR dynamic linewidth $\sim 1 \mathrm{MHz}$ [7]).

When no signal pre-emphasis was applied to the transmitter DS-DBR laser, (continuous) $\mathrm{CH} 1$ experienced significant cross channel interference during the time when the adjacent 

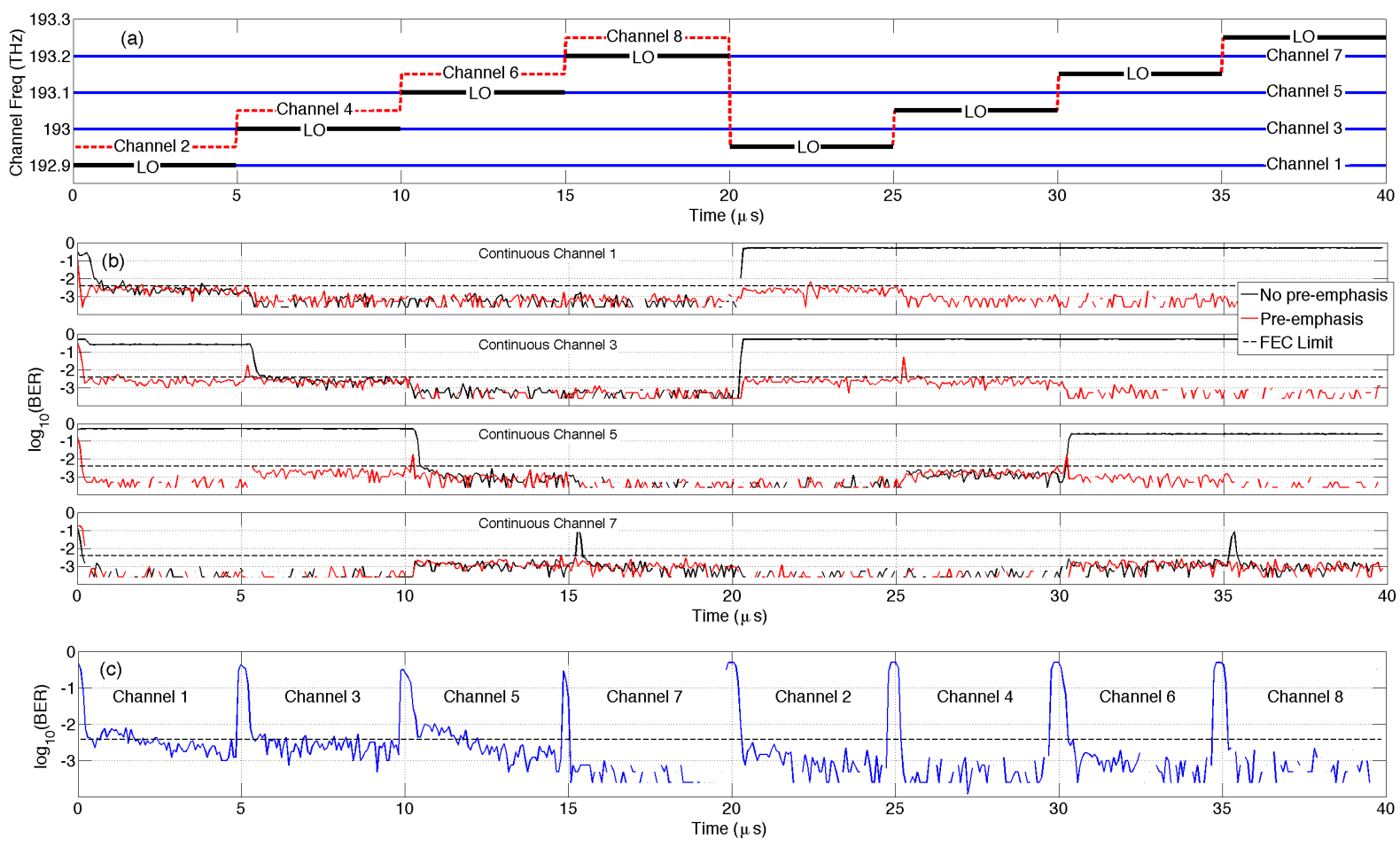

Fig. 3. (a) Channel switching map: two cycles of four dynamic $5 \mu$ s burst channels and four continuous channels. Also shown is sequentially switching LO, (b) differentially decoded BER of the four odd continuous channels with and without pre-emphasis of the transmit DS-DBR laser and (c) differentially decoded BER of all 8-channels under switched LO.

switching channel $(\mathrm{CH} 2)$ was present. The interference causes the CMA equalizer in the receiver to diverge, thus requiring a reset, which inevitably results in a burst of errors while the equalizer re-converges. Upon re-convergence a bit slip can also be experienced, which results in catastrophic bit errors (occurring after $20 \mu$ s for $\mathrm{CH} 1$ in Fig3. (b)).

When pre-emphasis was employed no cross channel interference was experienced, hence the receiver did not require a reset and the error bursts were mitigated. Similar performance was achieved for all four continuous channels. Fig. 3 (b) also shows that the BER of the continuous channels was degraded slightly when the adjacent switching channel was present, which was a result of linear cross talk arising from the limited extinction of the $50 \mathrm{GHz}$ interleaver that is used to decorrelate the $28 \mathrm{Gbaud}$ channels.

Fig. 3(c) illustrates the time resolved differentially decoded BER of all 8-channels when the LO sequentially switched through the continuous channels before wrapping back to cycle through the burst channels, as shown in Fig. 3(a). Pre-emphasis was applied to the transmitter DS-DBR laser and receiver was reset at the start of each incoming burst. The BER is shown to converge within 200ns for both the continuous and switched channels. The average OSNR penalty for all 8-channels relative to the ideal noise limited receiver was $3.4 \mathrm{~dB}$ at the FEC limit.

\section{Conclusions}

We have demonstrated the operation, over a $240 \mathrm{~km}$ uncompensated link, of a wavelength agile $112 \mathrm{~Gb} / \mathrm{s}$ coherent burst mode transceiver that employs commercially available DS-DBR lasers for fast wavelength switching at both the transmitter and the receiver in a $50 \mathrm{GHz}$ DWDM system. We have shown that SOA blanking and pre-emphasis is essential to avoid interference in systems where burst and continuous channels coexist and that differential detection is required to overcome the influence of the combined laser linewidths under switched operation.

\section{Acknowledgements}

We thank Oclaro Technology Limited for the supply of the DS-DBR lasers. R. Maher is supported by IRCSET, co-funded by Marie Curie Actions under FP7. B. Thomsen, S. Savory and D. Millar are supported by the EPRSC under Grants EP/D074088/1 and EP/G066159/1.

\section{References}

[1] A.K. kodi et al., J. Sel. Top. Quantum Electron. 17, p.384 (2011).

[2] J. Gripp et al., Proc. OFC'04, PDP6 (2004).

[3] J.E. Simsarian et al., Proc. OFC'10, PDPB5 (2010).

[4] A.J. Ward et al., J. of Quant. Electron. 11, 149 (1996).

[5] R. Maher, et al., Proc. OFC'12, JW2A.57 (2012).

[6] B.C. Thomsen et al., Opt. Express, 19, p.B770, (2011).

[7] R. Maher, et al., Opt. Express, 19, p.B313, (2011). 